\title{
Monitoring the Use of Telemonitor: A Resident- run Quality Improvement Initiative Decreases Inappropriate Use of Telemonitor in a Community Hospital
}

\author{
Tinashe Maduke ${ }^{1}$, Binish Qureshi ${ }^{1}$, Yohannes Goite ${ }^{1}$, Khushboo Gandhi ${ }^{1}$, Fadel Bofarrag ${ }^{1}$, Lin Liu ${ }^{1}$, \\ Miguel Suazo ${ }^{1}$, Sehrish Khan ${ }^{1}$, Samjhana Basnyat ${ }^{1}$, Suresh Dhital ${ }^{1}$, Hameem Kawsar ${ }^{2}$ \\ 1. Internal Medicine, St. Luke's Hospital, Chesterfield, USA 2. Internal Medicine/Hematology and Oncology, University \\ of Kansas Medical Center, Kansas City, USA
}

Corresponding author: Hameem Kawsar, hameem.kawsar@gmail.com

\section{Abstract \\ Background}

Cardiac telemetry is an important tool to detect life-threatening conditions in hospitalized patients but is used widely and inappropriately. We sought to assess current usage and improve the appropriate use of telemetry in a community hospital.

\section{Methods}

We conducted a quality improvement project on patients who were admitted on telemetry floors between January and March 2017 (pre-intervention). The indication(s) and duration of telemonitor use, event(s) recorded on telemonitor and outcome of the event(s) were documented. A six-month educational intervention was undertaken and the effect of intervention was assessed among patients admitted between December 2017 and February 2018 (post-intervention).

\section{Results}

In the pre-intervention group, 329 patients qualified for the study, with a median age of 78 years. The postintervention group had 383 qualified patients with a median age of 77 years. Mean duration of telemonitor use was four days in both groups. In the pre-intervention group, 54\% had class I, 32\% had class II, and $14 \%$ had class III indications. In post-intervention group, $46 \%$ had class I, $42 \%$ had class II, and $12 \%$ had class III indications. The educational intervention resulted in a trend towards less inappropriate use of telemetry, particularly in teaching service. Telemonitor events were recorded in 22 (7\%) of the pre-intervention patients and 13 (4\%) of the post-intervention group. Two patients died in the pre-intervention group and one in the post-intervention group from non-cardiac causes.

Received 11/25/2019

Review began 11/25/2019 Review ended 11/27/2019 Published 11/30/2019

\section{() Copyright 2019}

Maduke et al. This is an open access article distributed under the terms of the Creative Commons Attribution License CC-BY 3.0., which permits unrestricted use, distribution, and reproduction in any medium, provided the original author and source are credited.

\section{Conclusion}

Our results highlight that change in practice requires sustained education interventions.

Categories: Internal Medicine, Quality Improvement, Other

Keywords: telemonitor use, telemetry, quality improvement, choosing wisely, patient safety

\section{Introduction}

In 2004, American Heart Association (AHA) released guidelines on the use of telemonitor, and divided them into three classes: class I (telemonitor use indicated), class II (telemonitor may be beneficial) and class III (telemonitor not indicated) [1]. Studies have shown that telemonitor is often overused and used inappropriately. The Choosing Wisely ${ }^{\circledR}$ campaign, an American Board of Internal Medicine (ABIM) foundation initiative aimed at reducing waste and unnecessary tests in healthcare, recommended the use of guidelines to improve appropriate use of telemonitor in patients outside of ICU. Inappropriate use of telemetry has been widely studied and has shown to negatively affect patient care [2]. It can increase length of stay, cause alarm fatigue among providers, impact patient satisfaction, impact admission rates and cause backup in the emergency department [3,4]. It also adds extra cost to healthcare delivery [5]. The objective of this study was to evaluate the effectiveness of an educational intervention in reducing the inappropriate use of telemetry.

\section{Materials And Methods}

Study design and sampling 
We used a retrospective study looking at inpatient records of 712 patients in a 493-bed community teaching hospital in St Louis, MO. Inclusion criteria consisted non-ICU patients admitted on the telemetry floor or on a medical floor with remote cardiac monitoring. We excluded patients younger than 18 years of age, ICU patients, prior admission to ICU before transfer to telemetry floor, obstetrics and gynecology patients. We reviewed patients' chart who used cardiac monitor between January and March 2017 (pre-intervention) and December 2017 to February 2018 (post intervention). We selected patients who had cardiac monitoring ordered on admission and during admission. The study was approved by the hospital's institutional review board (IRB).

\section{Data collection}

We determined indications for cardiac monitoring from admission and progress notes. Duration on cardiac monitor was collected using order and cancellation dates and times from the electronic medical record. Data collection included principal diagnoses, age, gender, sex, days on telemetry, arrhythmias detected, rapid response/code blue events and transfer to ICU events. Data was gathered from St Luke's hospital electronic medical record system (Cerner) and recorded and analyzed on a secure Microsoft Excel and IBM SPSS.

\section{Educational intervention}

The intervention involved educational sessions with the hospitalists, Internal Medicine residents, cardiologists, emergency department physicians and nurse practitioners on the AHA cardiac monitoring guideline criteria for when it is indicated, beneficial and where it is not indicated. Each hospitalist, nurse practitioner and resident also received pocket cards with the criteria listed for them to use as a quick reference to decide when to order telemetry. Educational sessions were conducted as small group conferences, and structured and predefined reminder sessions for all participants. These sessions were conducted from August to November, 2017 with multiple reminder/refresher sessions.

\section{Outcomes}

The primary outcome was appropriateness of use of telemetry monitor as determined by the 2004 AHA guidelines. Secondary outcomes included duration on telemetry, events detected on cardiac monitor, occurrence of significant clinical events and death.

\section{Statistical analysis}

Continuous variables were analyzed using t-tests and ANOVA. Categorical data was analyzed using chisquare tests.

\section{Results}

\section{Patient characteristics}

In the pre-intervention group, 329 patients qualified for the study during January to March of 2017. Of these, 162 were in teaching service and 167 were in non-teaching service. The median age was 78 years and $48 \%$ of the patients were female. The data for post-intervention group was collected between December 2017 and February 2018, and 383 patients qualified for the study. The teaching service had 148 patients, while 245 patients were in non-teaching service. The median age was 77 years and $54 \%$ were female. Table 1 shows the characteristic of patients in both groups. The mean duration of telemonitor use was four days in both groups. Telemetry was continued until discharge in $93 \%$ of pre-intervention group and $91.6 \%$ of post intervention group $(\mathrm{p}=0.52)$. The main reasons for telemetry use were acute coronary syndromes, syncope, stroke/transient ischemic attack, decompensated congestive heart failure, atrial fibrillation and electrolyte abnormalities (Figure 1). 


\section{Cureus}

\begin{tabular}{|c|c|c|c|}
\hline & Pre-intervention & Post-intervention & p-value \\
\hline Sample size & 329 & 383 & \\
\hline Teaching/non-teaching & 162/167 & $148 / 235$ & \\
\hline Age (median, range) & $78(34-100)$ & $77(19-81)$ & 0.901 \\
\hline Gender, female (\%) & $158(48 \%)$ & 208 (54.3\%) & 0.157 \\
\hline \multicolumn{4}{|l|}{ AHA classes of indication for telemetry use } \\
\hline 1 & $179(54.4 \%)$ & 176 (46.0\%) & 0.025 \\
\hline II & 105 (31.9\%) & 162 (42.3\%) & 0.004 \\
\hline III & $45(13.7 \%)$ & $45(11.7 \%)$ & 0.42 \\
\hline Days on cardiac monitor (mean) & 4.03 & 4.05 & 0.928 \\
\hline Cardiac monitor used until discharge & $300(93.2 \%)$ & 351 (91.6\%) & 0.518 \\
\hline Events on monitor & $22(6.7 \%)$ & $14(3.7 \%)$ & 0.066 \\
\hline Actions taken for monitored event(s) & 6 (27.3\%) & $4(26.7 \%)$ & 0.967 \\
\hline Deaths & 2 & 1 & 0.476 \\
\hline
\end{tabular}

TABLE 1: Patient and event characteristics.

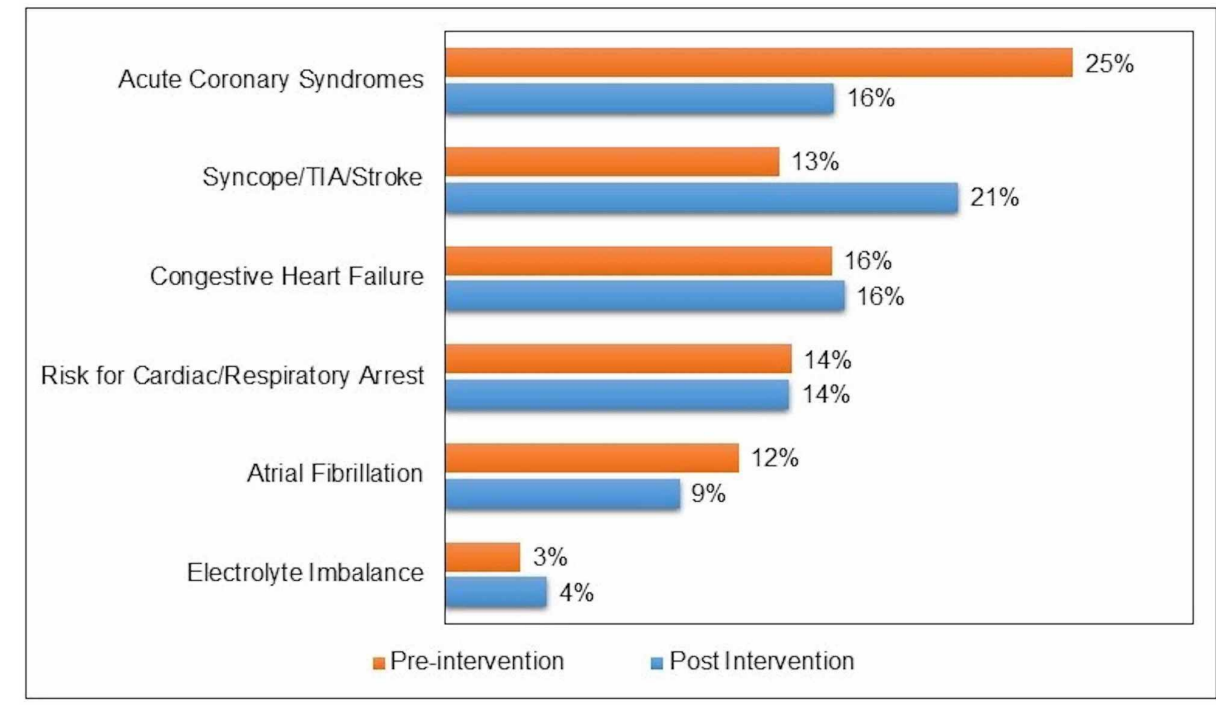

FIGURE 1: Common reasons for using telemetry.

\section{Use of telemonitor according to AHA indications}

In the pre-intervention group, $54 \%$ had class I (indicated), $32 \%$ had class II (beneficial), and $14 \%$ had class III (not indicated) indications. In post-intervention group, $46 \%$ had class I, $42 \%$ had class II, and $12 \%$ had class III indications. As such there was a $2 \%$ drop in the inappropriate use (Class III), but this was not statistically significant (Figure 2). The educational intervention resulted in a trend towards less inappropriate ordering of telemetry, particularly in teaching service over successive months (Figure 3). This effect was more pronounced in the teaching service which involved residents as compared to non-teaching service (Figure 4). 


\section{Cureus}

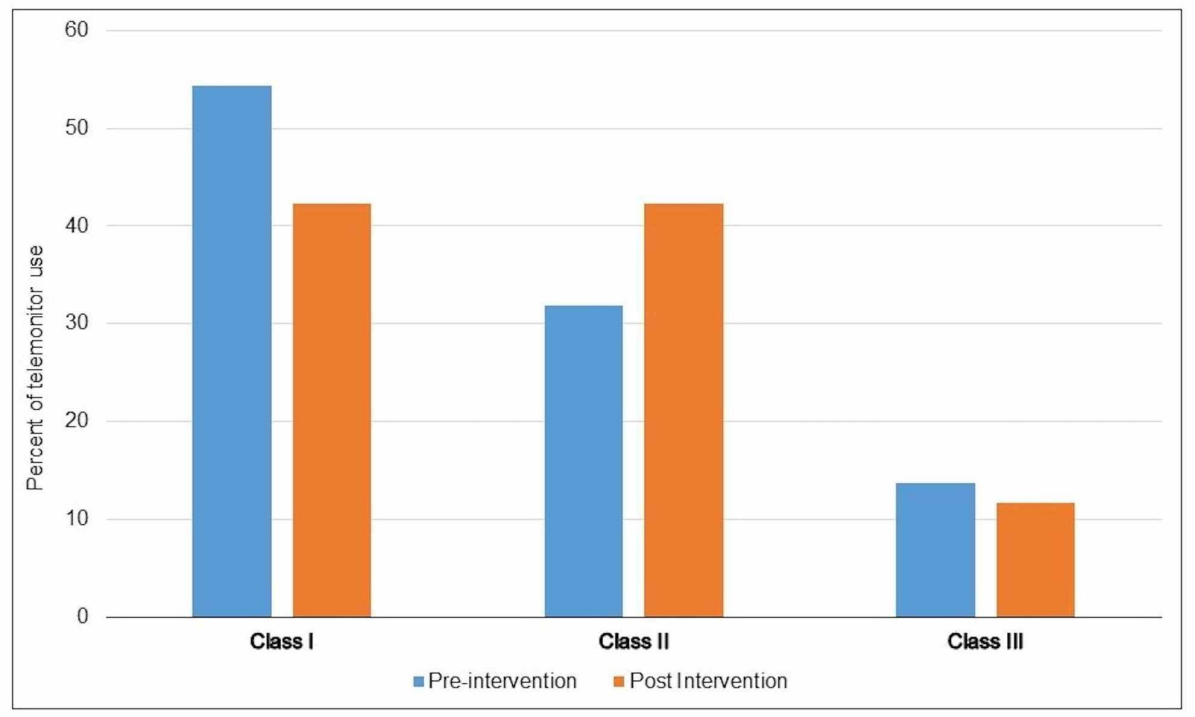

FIGURE 2: Use of telemonitor according to AHA indication (Class I: indicated, Class II: beneficial, and Class III: not indicated).

AHA: American Heart Association

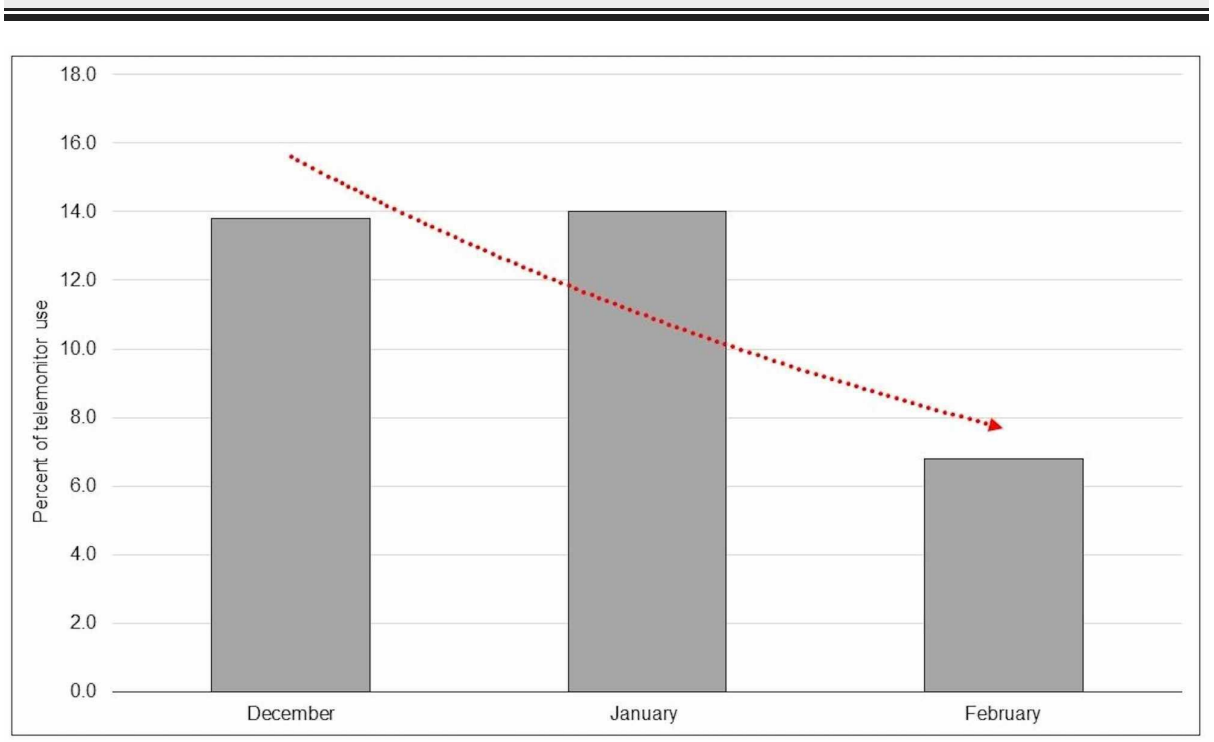

FIGURE 3: Declining trend of AHA class III (not indicated) use of telemetry in subsequent months in post-intervention period (trend line in red).

AHA: American Heart Association 


\section{Cureus}

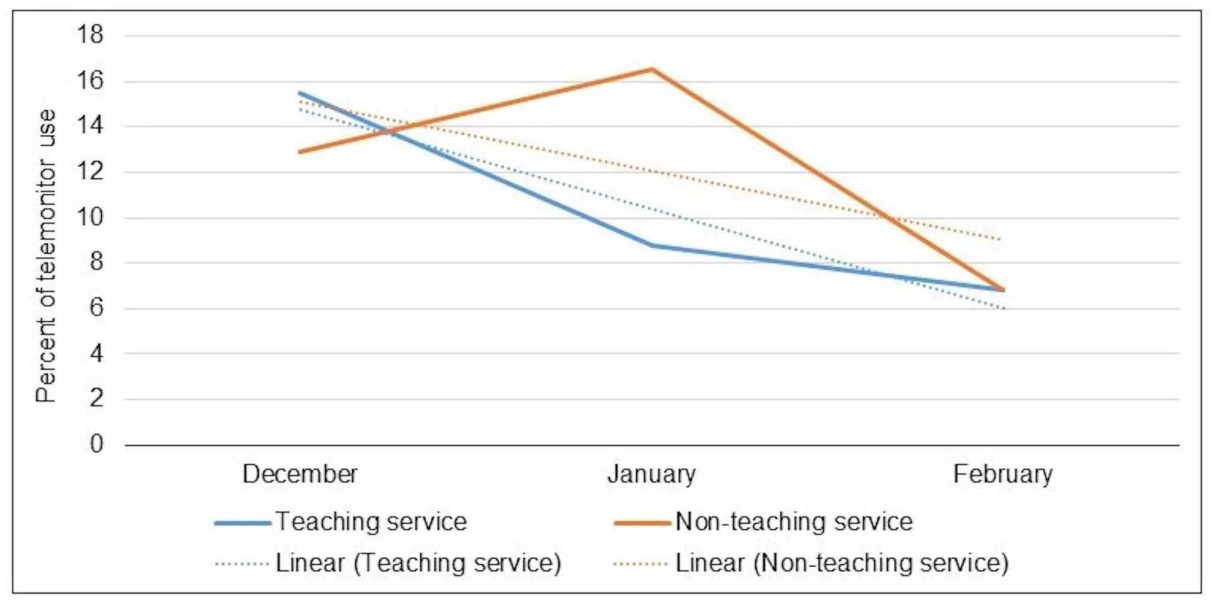

FIGURE 4: Trend of inappropriate use (AHA class III, not indicated) of telemonitor in teaching and non-teaching service.

AHA: American Heart Association

\section{Telemonitor events}

In the pre-intervention group, 22 events ( $7 \%$ of the sample) were detected on the telemonitor. Of these, actions were taken on six events (27\%). In the post intervention group, there were 13 events (4\%), and actions were taken on four events (27\%). Actions included changes, or starting new medications, cardioversion or transfer to the intensive care unit (ICU). The commonest events picked up on the telemonitor were atrial fibrillation, non-sustained ventricular tachycardia, atrial flutter, bradycardia, torsade de pointes, and multifocal atrial tachycardia (Figure 5). Two patients died in the pre-intervention group and one in the post-intervention group from non-cardiac causes.

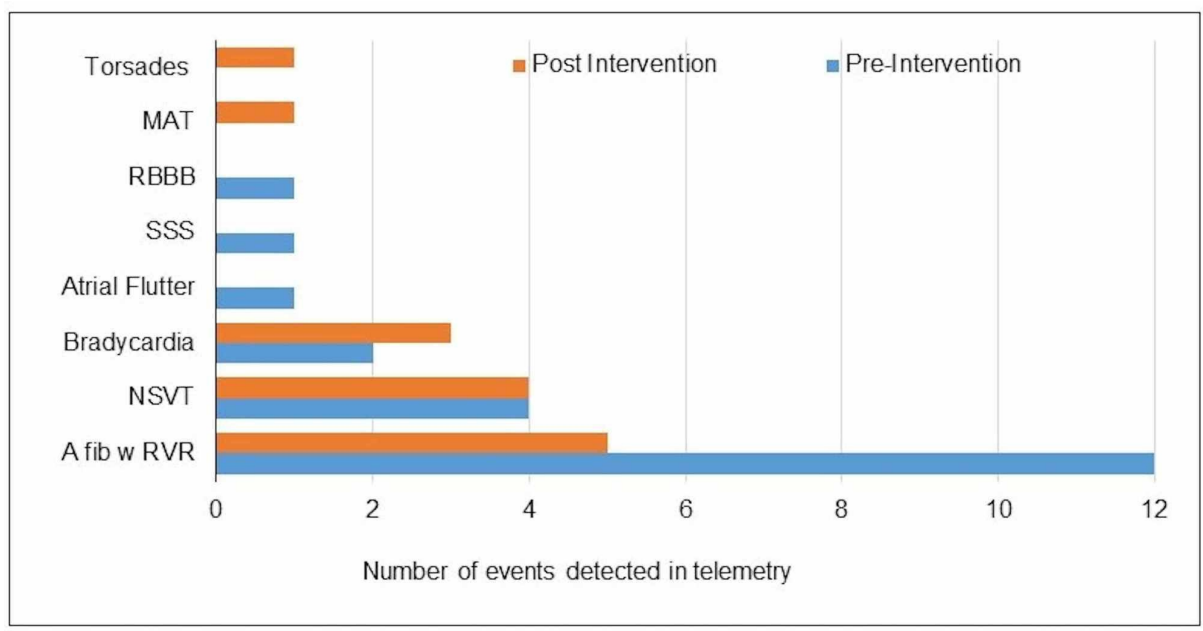

\section{FIGURE 5: Cardiac events detected in the telemonitor.}

MAT: Multifocal atrial tachycardia; RBBB: Right bundle branch block; SSS: Sick sinus syndrome; NSVT: Nonsustained ventricular tachycardia; A fib w RVR: Atrial fibrillation with rapid ventricular response.

\section{Reason for inappropriate telemetry use}

Viral respiratory infections accounted for the largest share of inappropriate telemetry use (22\%). Other reasons included pneumonia (10\%), urinary tract infections (9\%), chronic stable atrial fibrillation (7\%), COPD exacerbation, hypertension, fever of unknown origin and cellulitis which individually accounted for $4 \%$ (Figure 6). 


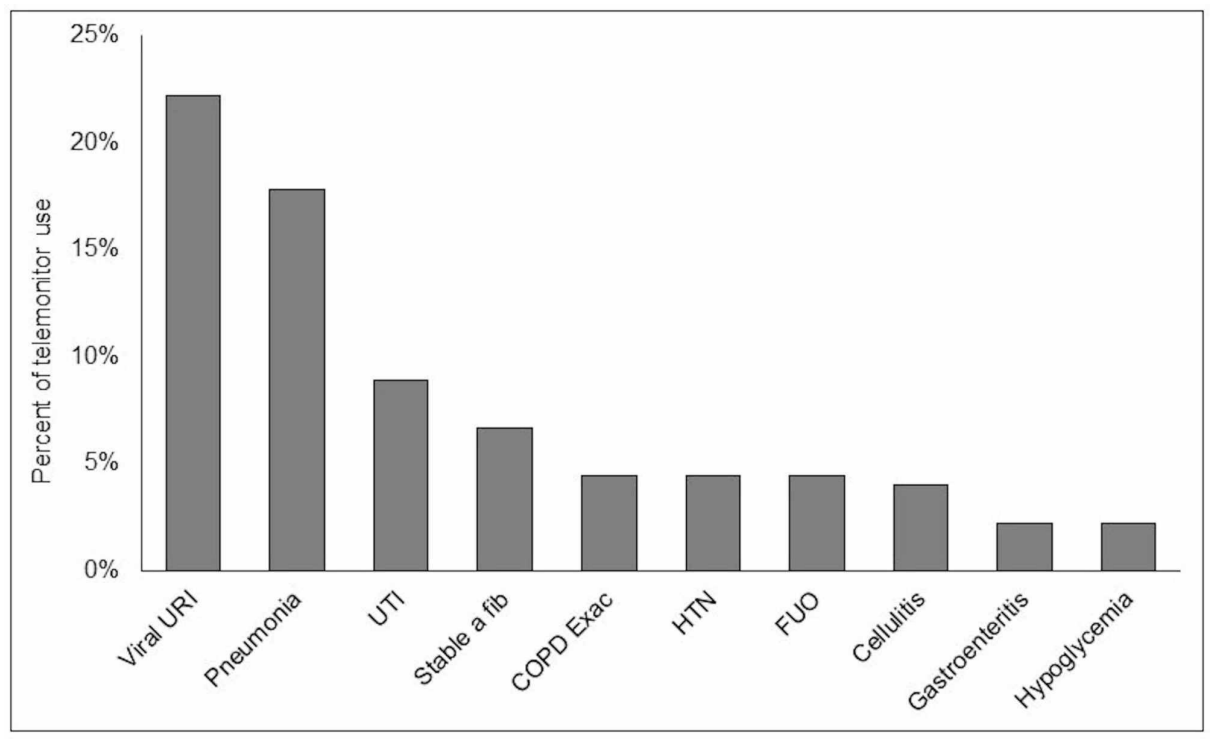

FIGURE 6: Common reasons for inappropriate use of telemonitor (AHA class III).

URI: Urinary tract infection; a fib: Atrial fibrillation; COPD exac: Chronic obstructive pulmonary disease exacerbation; HTN: Hypertension; FUO: Fever of unknown origin; AHA: American Heart Association.

\section{Discussion}

This resident-run quality improvement project highlighted a previously noted problem in the provision of telemetry use when it is not clinically indicated. The Society for Hospital Medicine has made reducing the unnecessary use of telemetry a top priority in the Choosing Wisely campaign. Our study showed inappropriate utilization of telemonitor ranges between $12 \%-14 \%$. This at first glance may seem reasonable but is offset by the significant cost associated with this resource. Inappropriate telemetry use rate in our study is lower than those noted in other hospitals of similar size where it was as high as $20-30 \%$ [6]. The use of guideline-based interventions has shown promise in reducing the inappropriate use of telemetry [7]. This study's design to use guidelines to order telemonitor showed some modest improvement in the appropriateness of telemonitor use, particularly in patients on the teaching service. It seems that involvement of residents in the intervention process was associated with greater improvement. The probable reasons for this improvement include increased enthusiasm in the project run by fellow residents, and keenness to learn new information and use it appropriately.

One key finding was that most patients who were admitted to the telemetry unit tended to stay on cardiac monitor regardless of resolution of the indication or having no indication at the outset. More than $90 \%$ of patients remained on telemetry throughout their admission. This signifies that once a patient is diagnosed with possible cardiac or neurological event, they are likely to be monitored with telemonitor throughout their hospitalization. The educational intervention did not result in a significant change in the telemonitor discontinuation rates. Further interventions likely need to be incorporated to remind providers of the need to discontinue telemetry at the earliest appropriate opportunity as clinically indicated. A study by Rizvi et al. showed that incorporating pop-ups in the electronic medical system for discontinuing telemetry reduced overuse of telemetry by $37 \%$ [8]. Such an intervention or the use of expiration dates with initial order of telemetry, which currently does not exist in our hospital, may be helpful in stopping telemetry when no longer indicated.

This study further illustrated that only few events were detected on telemetry, and an even smaller number of these events required change of management. Clinically significant events were only noted in 3-7\% of patients and only a third of these events resulted in changes in medications or transfer to a higher level of care such as transfer to the intensive care unit. This low yield from telemetry and rare subsequent transfer to ICU has been documented in other studies [9-12]. Our results also showed the arrhythmias were detected only in the patients who had appropriate indication for use of telemetry, further reinforcing the need to restrict telemetry use in clinical- and guideline-directed indicated cases. Further exploration into incorporating an electronic order system, where providers indicate the reason for ordering telemetry, can result in further improvement in the ordering pattern. It appears that addressing this problem will likely require a multi-pronged approach, incorporating a sustained educational intervention, and indication-based electronic order system.

Analysis of the main reasons for inappropriate telemetry use showed that a significant proportion of 
patients had telemetry ordered for respiratory infections, chronic stable atrial fibrillation, urinary tract infections and exacerbations of obstructive lung disease. The median age of patients in our study was 77 years, and thus the concern for sick elderly patients likely prompted admission for closer observation with use of telemonitor. However, the yield of telemonitor use is typically low in such patients. A study of telemetry on geriatric patients with low risk for in-hospital coronary events showed that none of the patients admitted to telemetry developed clinically significant events [13].

Our study was limited in that the duration of post intervention evaluation period was short. Three months was likely not adequate to evaluate the sustained effect of the intervention. Determination of the appropriateness of telemetry use was based on what was documented in the charts. It is possible that the ordering providers may have had other reasons unclear to the chart reviewer(s) which possibly could have created a bias in the result. The sample size was also relatively small and possibly could have overestimated or underestimated the outcomes. Moreover, the retrospective nature of the study is vulnerable to selection bias. One of the limitations is that the study population was not homogeneous between pre- and postintervention group. As a result, for class II indication, there was 105 (32\%) patients in pre-intervention group and 162 (42\%) patients in post-intervention group. This difference in sample size in two groups was significant, which might have affected the interpretation of data for class II indication. Nonetheless, this was a resident-run quality improvement project to increase awareness among house staffs as well as to improve guideline and evidence-based use of telemonitor in hospitalized patients.

\section{Conclusions}

Our results highlight that emphasizing guideline-based utilization of the telemonitor in non-ICU patients can aid in ensuring appropriate use of this resource. However, the effect of the educational campaign was modest and further studies with longer post-intervention follow-up and combining with electronic medical system interventions need to be assessed to see greater changes in ordering patterns of providers.

\section{Additional Information \\ Disclosures}

Human subjects: Consent was obtained by all participants in this study. IRB of St. Luke's Hospital issued approval Not applicable. It is a retrospective chart-review and was approved by the IRB. Animal subjects: All authors have confirmed that this study did not involve animal subjects or tissue. Conflicts of interest: In compliance with the ICMJE uniform disclosure form, all authors declare the following: Payment/services info: All authors have declared that no financial support was received from any organization for the submitted work. Financial relationships: All authors have declared that they have no financial relationships at present or within the previous three years with any organizations that might have an interest in the submitted work. Other relationships: All authors have declared that there are no other relationships or activities that could appear to have influenced the submitted work.

\section{Acknowledgements}

We would like to thank Dr. Fred Balis and the Residency Program of Internal Medicine for supporting this resident-run quality improvement project.

\section{References}

1. Drew BJ, Califf RM, Funk M, et al.: Practice standards for electrocardiographic monitoring in hospital settings: an American Heart Association scientific statement from the Councils on Cardiovascular Nursing, Clinical Cardiology, and Cardiovascular Disease in the Young: endorsed by the International Society of Computerized Electrocardiology and the American Association of Critical-Care Nurses. Circulation. 2004, 110:2721-2746. 10.1161/01.CIR.0000145144.56673.59

2. Dhillon SK, Rachko M, Hanon S, Schweitzer P, Bergmann SR: Telemetry monitoring guidelines for efficient and safe delivery of cardiac rhythm monitoring to noncritical hospital inpatients. Crit Pathw Cardiol. 2009, 8:125-126. 10.1097/HPC.0b013e3181b6ef41

3. Knight BP, Pelosi F, Michaud GF, Strickberger SA, Morady F: Clinical consequences of electrocardiographic artifact mimicking ventricular tachycardia. N Engl J Med. 1999, 341:1270-1274. 10.1056/NEJM199910213411704

4. Wallis L: Alarm fatigue linked to patient's death. Am J Nurs. 2010, 110:16. 10.1097/01.NAJ.0000383917.98063.bd

5. Najafi N, Auerbach A: Use and outcomes of telemetry monitoring on a medicine service . Arch Intern Med. 2012, 172:1349-1350. 10.1001/archinternmed.2012.3163

6. Benjamin EM, Klugman RA, Luckmann R, Fairchild DG, Abookire SA: Impact of cardiac telemetry on patient safety and cost. Am J Manag Care. 2013, 19:225-232. 10.1183/16000617.0116-2016

7. Dressler R, Dryer MM, Coletti C, Mahoney D, Doorey AJ: Altering overuse of cardiac telemetry in nonintensive care unit settings by hardwiring the use of American Heart Association guidelines. JAMA Intern Med. 2014, 174:1852-1854. 10.1001/jamainternmed.2014.4491

8. Rizvi W, Munguti CM, Mehta J, Kallail KJ, Youngman D, Antonios S: Reducing over-utilization of cardiac telemetry with pop-ups in an electronic medical record system. Cureus. 2017, 9:1282. 10.7759/cureus.1282

9. Curry JP, Hanson CW 3rd, Russell MW, Hanna C, Devine G, Ochroch EA: The use and effectiveness of electrocardiographic telemetry monitoring in a community hospital general care setting. Anesth Analg. 


\section{Cureus}

2003, 97:1483-1487. 10.1213/01.ane.0000081720.49358.53

10. Estrada CA, Rosman HS, Prasad NK, Battilana G, Alexander M, Held AC, Young MJ: Evaluation of guidelines for the use of telemetry in the non-intensive-care setting. J Gen Intern Med. 2000, 15:51-55. 10.1046/j.15251497.2000.112188.x

11. Sivaram CA, Summers JH, Ahmed N: Telemetry outside critical care units: patterns of utilization and influence on management decisions. Clin Cardiol. 1998, 21:503-505. 10.1002/clc.4960210709

12. Snider A, Papaleo M, Beldner S, Park C, Katechis D, Galinkin D, Fein A: Is telemetry monitoring necessary in low-risk suspected acute chest pain syndromes?. Chest. 2002, 122:517-523. 10.1378/chest.122.2.517

13. Saleem MA, McClung JA, Aronow WS, Kannam H: Inpatient telemetry does not need to be used in the management of older patients hospitalized with chest pain at low risk for in-hospital coronary events and mortality. J Gerontol A Biol Sci Med Sci. 2005, 60:605-606. 10.1093/gerona/60.5.605 